\title{
Structure galoisienne et corps de classes de rayon de conducteur 2
}

\author{
par
}

\author{
E. J. Gómez Ayala (Bilbao)
}

Si $F$ est un corps de nombres, on note $\mathfrak{O}_{F}$ son anneau d'entiers. Soit $K$ un corps quadratique imaginaire, $H_{K}$ le corps de classes de Hilbert de $K, \mathfrak{p}$ un idéal premier de $\mathfrak{O}_{K}$ et $K(\mathfrak{p})$ le corps de classes de rayon de $K$ de conducteur $\mathfrak{p}$. L'extension $\left(K(\mathfrak{p}) / H_{K}\right)$ peut être considérée comme un analogue elliptique de l'extension cyclotomique $\left(\mathbb{Q}\left(\zeta_{p}\right) / \mathbb{Q}\right)$ pour un nombre premier $p$. Mais contrairement au cas cyclotomique, c'est un problème non résolu de savoir s'il existe ou non une base normale de $\mathfrak{O}_{K(\mathfrak{p})}$ sur $\mathfrak{O}_{H_{K}}$.

Soit $K=\mathbb{Q}(\sqrt{-d})$ avec $d=11,19,43,67$ ou 163 et soit $K(2)$ le corps de classes de rayon de $K$ de conducteur 2 . Le corps $K(2)$ est une extension cubique et modérément ramifiée de $K$. Le but de cet article est de montrer l'existence d'une base normale de $\mathfrak{O}_{K(2)}$ sur $\mathfrak{O}_{K}$ et de faire sa construction explicite. Plus précisément, on va démontrer le théorème suivant :

THÉORÈme. Les racines du polynôme $X^{3}-X^{2}+X+1$ (resp. $X^{3}-X^{2}+$ $\left.X-163, X^{3}-X^{2}+X-447, X^{3}-X^{2}+X+663, X^{3}-X^{2}+X-15\right)$ constituent une base normale de $\mathfrak{O}_{K(2)}$ sur $\mathfrak{O}_{K}$ pour $K=\mathbb{Q}(\sqrt{-11})$ (resp. $K=\mathbb{Q}(\sqrt{-19}), K=\mathbb{Q}(\sqrt{-43}), K=\mathbb{Q}(\sqrt{-67}), K=\mathbb{Q}(\sqrt{-163}))$.

Je remercie R. Schertz ([10]), qui m'a proposé d'examiner ces extensions du point de vue de la structure galoisienne. La démonstration du théorème (§2 et $\S 3)$ repose sur un critère générale d'existence de base normale d'entiers dans les extensions de Kummer de degré premier qui a été démontré dans [3]. Le point de vue de Schertz, plus dans l'esprit explicite du Jugendtraum de Kronecker, est exposé dans le $\S 4$.

1. Préliminaires. Les deux lemmes suivants sont faciles à démontrer.

Lemme 1.1. Soit $k \subseteq L \subseteq M$ une tour d'extensions finies de corps de nombres telles que $(M / k)$ et $(L / k)$ sont galoisiennes. Si a engendre une base normale de $\mathfrak{O}_{M}$ sur $\mathfrak{O}_{k}$, alors $\operatorname{Tr}_{M / L}(a)$ engendre une base normale de $\mathfrak{O}_{L}$ $\operatorname{sur} \mathfrak{O}_{k}$. 
Voir [8], Chap. IX, §3, Theorem 3.4.

LEMME 1.2. Soit $L$ une extension galoisienne finie d'un corps de nombres $k, F$ une extension finie de $k$ et $E=F L$. On suppose que $L \cap F=k$ et que les discriminants $D_{L / k}$ et $D_{F / k}$ sont premiers entre eux. Si a engendre une base normale de $\mathfrak{O}_{L}$ sur $\mathfrak{O}_{k}$, alors a engendre une base normale de $\mathfrak{O}_{E}$ sur $\mathfrak{O}_{F}$.

C'est une conséquence du fait que $\mathfrak{O}_{F L}=\mathfrak{O}_{F} \mathfrak{O}_{L}$ sous les hypothèses du lemme ([6], Chap. III, §3, Proposition 17).

Soit maintenant $K=\mathbb{Q}(\sqrt{-d})$ avec $d=11,19,43,67$ ou $163, L$ une extension cubique de $K, F=K(\omega)$ (avec $\omega=\exp (2 \pi i / 3)$ ) et $E=F L$ le composé de $F$ et $L$.

Proposition 1.3. Soit $a \in \mathfrak{O}_{L}$. Alors a engendre une base normale de $\mathfrak{O}_{L}$ sur $\mathfrak{O}_{K}$ si et seulement si a engendre une base normale de $\mathfrak{O}_{E}$ sur $\mathfrak{O}_{F}$.

On a un diagramme de corps et extensions de corps :

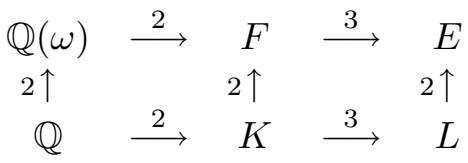

Supposons que $a$ engendre une base normale de $\mathfrak{O}_{L}$ sur $\mathfrak{O}_{K}$; ceci implique que l'extension $(L / K)$ est modérément ramifiée et par conséquent les discriminants $D_{L / K}$ et $D_{F / K}$ sont premiers entre eux. Puisque $L \cap F=K$, l'élément $a$ engendre une base normale de $\mathfrak{O}_{E}$ sur $\mathfrak{O}_{F}$, d'après le lemme 1.2.

Réciproquement, soit $\operatorname{Gal}(E / F)=\left\{1, \sigma, \sigma^{2}\right\}$ et supposons que $a$ engendre une base normale $\left\{a, a^{\sigma}, a^{\sigma^{2}}\right\}$ de $\mathfrak{O}_{E}$ sur $\mathfrak{O}_{F}$. On a $K \cap \mathbb{Q}(\omega)=\mathbb{Q}$ et les discriminants $D_{K / \mathbb{Q}}$ et $D_{\mathbb{Q}(\omega) / \mathbb{Q}}$ sont premiers entre eux car l'idéal $3 \mathbb{Z}$ ne se ramifie pas dans l'extension $(K / \mathbb{Q})$. Soit $\operatorname{Gal}(F / K) \simeq \operatorname{Gal}(\mathbb{Q}(\omega) / \mathbb{Q})=$ $\{1, \tau\}$ (avec $\omega^{\tau}=\omega^{2}$ ); puisque $\omega$ engendre une base normale $\left\{\omega, \omega^{\tau}\right\}$ de $\mathbb{Z}[\omega]$ sur $\mathbb{Z}, \omega$ engendre aussi une base normale de $\mathfrak{O}_{F}$ sur $\mathfrak{O}_{K}$, d'après le lemme 1.2. Soit $\varrho$ le seul élément de $\operatorname{Gal}(E / K)$ tel que $\omega^{\varrho}=\omega^{\tau}$ et $\varrho$ est l'identité sur $L$, de sorte que $\operatorname{Gal}(E / K)=\left\{1, \sigma, \sigma^{2}, \varrho, \sigma \varrho, \sigma^{2} \varrho\right\}$. On sait que les produits $\left\{a \omega, a^{\sigma} \omega, a^{\sigma^{2}} \omega, a \omega^{\tau}, a^{\sigma} \omega^{\tau}, a^{\sigma^{2}} \omega^{\tau}\right\}$ constituent une base de $\mathfrak{O}_{E}$ comme $\mathfrak{O}_{K}$-module, mais en fait cette base est une base normale, car ces éléments sont les conjugués du produit $a \omega$ sous l'action de $\operatorname{Gal}(E / K)$ compte tenu du fait que $a^{\varrho}=a$, puisque $a \in \mathfrak{O}_{L}$. On en déduit que $a \omega$ engendre une base normale de $\mathfrak{O}_{E}$ sur $\mathfrak{O}_{K}$. D'après le lemme 1.1, $\operatorname{Tr}_{E / L}(a \omega)$ engendre une base normale de $\mathfrak{O}_{L}$ sur $\mathfrak{O}_{K}$. Mais $\operatorname{Tr}_{E / L}(a \omega)=-a$ et par conséquent $a$ engendre une base normale de $\mathfrak{O}_{L}$ sur $\mathfrak{O}_{K}$.

On sait que $\mathfrak{O}_{K}$ est un anneau principal. Soit $K(2)$ le corps de classes de rayon de $K$ de conducteur 2; puisque 2 est inerte dans $K$, on sait que $K(2)$ est une extension cubique de $K$ ([7], Chap. VIII, $\S 1$, Theorem 7). Le 
seul idéal premier de $K$ qui se ramifie dans l'extension $(K(2) / K)$ est $2 \mathfrak{O}_{K}$. Par conséquent, l'extension $(K(2) / K)$ est modérément ramifiée.

On connait un critère explicite pour l'existence d'une base normale d'entiers dans une extension de Kummer cubique ([3], Proposition 2.13) :

Proposition 1.4. Soit E/F une extension de Kummer cubique. Alors $\mathfrak{O}_{E}$ possède une base normale sur $\mathfrak{O}_{F}$ si et seulement s'il existe $\alpha \in \mathfrak{O}_{E}$ tel que $\alpha^{3} \in \mathfrak{O}_{F}, E=F(\alpha), \alpha \equiv 1 \bmod (1-\omega), \alpha^{3} \mathfrak{O}_{F}=\mathfrak{a b}^{2}$, où $\mathfrak{a}$ et $\mathfrak{b}$ sont des idéaux premiers entre eux et sans facteurs carrés, et $\mathfrak{b}$ est un idéal principal avec un générateur $x$ tel que $x \equiv 1 \bmod 3$. Dans ce cas, $a=(1 / 3)\left(1+\alpha+\left(\alpha^{2} / x\right)\right)$ engendre une base normale de $\mathfrak{O}_{E}$ sur $\mathfrak{O}_{F}$.

Soit dorénavant $F=K(\omega)$ et $E=K(2) F$ le composé de $K(2)$ et $F$. D'après les propositions 1.3 et 1.4 , il est donc naturel d'étudier d'abord s'il existe ou non une base normale de $\mathfrak{O}_{E}$ sur $\mathfrak{O}_{F}$, et la réponse à cette question étant positive, comme l'on montre dans le $\S 2$, d'essayer ultérieurement d'en tirer une base normale de $\mathfrak{O}_{L}$ sur $\mathfrak{O}_{K}$, ce qui est fait dans le $\S 3$.

2. Étude de l'extension de Kummer $(E / F)$. On va montrer qu'il existe une base normale de $\mathfrak{O}_{E}$ sur $\mathfrak{O}_{F}$.

Proposition 2.1. Soit $K=\mathbb{Q}(\sqrt{-d})$ avec $d=11,19,43,67$ ou 163 , $F=K(\sqrt{-3})$ et $E=K(2) F$ le composé de $K(2)$ et $F$. Alors il existe une base normale de $\mathfrak{O}_{E}$ sur $\mathfrak{O}_{F}$.

Examinons les propriétés de l'extension $(E / F)$. On a un diagramme de corps et extensions de corps :

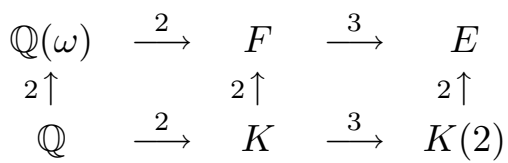

Les seuls idéaux premiers de $F$ qui peuvent se ramifier dans l'extension $(E / F)$ sont les idéaux premiers de $F$ qui sont au-dessus de $2 \mathfrak{O}_{K}$. Puisque l'extension $(F / \mathbb{Q})$ n'est pas cyclique, $2 \mathfrak{O}_{F}$ n'est pas un idéal premier de $\mathfrak{O}_{F}$; il n'est pas non plus ramifié sur $\mathbb{Q}$, car l'idéal $2 \mathbb{Z}$ ne se ramifie pas dans $(\mathbb{Q}(\omega) / \mathbb{Q})$ ni dans $(K / \mathbb{Q})$. On a donc $2 \mathfrak{O}_{F}=\mathfrak{p q}$ avec $\mathfrak{p}$ et $\mathfrak{q}$ des idéaux premiers distincts de $\mathfrak{O}_{F}$ et tels que $\mathfrak{q}=\mathfrak{p}^{\tau}$, où $\tau$ est le seul élément non trivial de $\operatorname{Gal}(F / K)$. On sait que $\mathfrak{O}_{F}$ est principal ([2], Case I); on peut déterminer facilement des générateurs pour ces idéaux premiers de la forme $(1 / 2)(a \sqrt{-3}+b \sqrt{-d})$ avec $a, b \in \mathbb{Z}$ en résolvant l'équation $3 a^{2}-d b^{2}= \pm 8$. On pose $\pi=(1 / 2)(\sqrt{-3}+\sqrt{-11})$ (resp. $\pi=(1 / 2)(3 \sqrt{-3}+\sqrt{-19}), \pi=$ $(1 / 2)(19 \sqrt{-3}+5 \sqrt{-43}), \pi=(1 / 2)(5 \sqrt{-3}+\sqrt{-67}), \pi=(1 / 2)(-715 \sqrt{-3}+$ $97 \sqrt{-163})$ ) pour $d=11$ (resp. pour $d=19, d=43, d=67, d=163$ ), $\mathfrak{p}=\pi \mathfrak{O}_{F}$ et $\mathfrak{q}=\pi^{\tau} \mathfrak{O}_{F} ;$ on remarque que $\pi \pi^{\tau}=-2$ pour $d=11$ et $\pi \pi^{\tau}=2$ pour les autres valeurs de $d$. 
En fait $E=F(2)$, le corps de classes de rayon de $F$ de conducteur 2 . En effet, $K(2) \subseteq F(2)$ par la théorie du corps de classes et par conséquent $E \subseteq F(2)$. Puisque $\mathfrak{O}_{F}$ est principal, le groupe de Galois de $F(2)$ sur $F$ est isomorphe à $\left(\mathfrak{O}_{F} / 2 \mathfrak{O}_{F}\right)^{*} / \operatorname{Im}\left(\mathfrak{O}_{F}^{*}\right)$. Le groupe $\left(\mathfrak{O}_{F} / 2 \mathfrak{O}_{F}\right)^{*}$ est un groupe à $\Phi(2)=\Phi(\mathfrak{p q})=\Phi(\mathfrak{p}) \Phi(\mathfrak{q})=9$ éléments et l'image du groupe engendré par $-\omega$ dans $\left(\mathfrak{O}_{F} / 2 \mathfrak{O}_{F}\right)^{*}$ est à trois éléments. On en déduit que $[F(2): F] \leq 3$ et par conséquent $E=F(2)$.

Le corps $E$ est une extension galoisienne cubique de $F$ dans laquelle les seuls idéaux premiers de $F$ qui se ramifient sont $\mathfrak{p}$ et $\mathfrak{q}$. De plus, $E$ est la seule extension galoisienne cubique de $F$ qui possède cette propriété. En effet, soit $E^{\prime}$ une extension galoisienne cubique de $F$ dans laquelle les seuls idéaux premiers de $F$ qui se ramifient sont $\mathfrak{p}$ et $\mathfrak{q}$; par la théorie du corps de classes, il existe $i \geq 1$ et $j \geq 1$ tels que $E^{\prime} \subseteq F\left(\mathfrak{p}^{i} \mathfrak{q}^{j}\right)$. Mais le groupe de Galois de $F\left(\mathfrak{p}^{i} \mathfrak{q}^{j}\right)$ sur $F$ est isomorphe à $\left(\mathfrak{O}_{F} / \mathfrak{p}^{i} \mathfrak{q}^{j}\right)^{*} / \operatorname{Im}\left(\mathfrak{O}_{F}^{*}\right)$, un groupe abélien à $\Phi\left(\mathfrak{p}^{i} \mathfrak{q}^{j}\right)=\Phi\left(\mathfrak{p}^{i}\right) \Phi\left(\mathfrak{q}^{j}\right)=4^{i+j-2} 9$ éléments; puisque l'image du groupe engendré par $-\omega$ dans ce groupe est d'ordre 3 ou 6 , il n'existe qu'un seul corps $L$ tel que $F \subseteq L \subseteq F\left(\mathfrak{p}^{i} \mathfrak{q}^{j}\right)$ et $[L: F]=3$; puisque $F \subseteq E=F(2) \subseteq F\left(\mathfrak{p}^{i} \mathfrak{q}^{j}\right)$ et $[E: F]=3$, on a donc $L=E^{\prime}=E$.

On va montrer qu'il existe $t \in \mathfrak{O}_{F}^{*}$ tel que $2 \pi t \equiv 1 \bmod (1-\omega)^{3}$; ce fait impliquera, d'après le critère de ramification de Hecke pour les extensions de Kummer de degré premier ([4], Chap. V, §39, Theorems 118 et 119) que $F\left((2 \pi t)^{1 / 3}\right)$ est une extension galoisienne cubique de $F$ dans laquelle les seuls idéaux premiers de $F$ qui se ramifient sont $\mathfrak{p}$ et $\mathfrak{q}$ et par conséquent que $E=F\left((2 \pi t)^{1 / 3}\right)$.

En effet, soit $k=\mathbb{Q}(\sqrt{3 d})$ le sous-corps quadratique réel de $F$. D'après les tables numériques réalisées Bordeaux, $u=23+4 \sqrt{33}$ (resp. $u=151+20 \sqrt{57}$, $u=16855+1484 \sqrt{129}, u=515095+36332 \sqrt{201}, u=7592629975+$ $343350596 \sqrt{489}$ ) est l'unité fondamentale de $k$ pour $d=11$ (resp. pour $d=$ $19, d=43, d=67, d=163)$. Un théorème de Kubota ([5], §3, Satz 2) affirme que si $v^{2}=-u$, alors $v$ est une unité fondamentale de $F$; on obtient ainsi que $v=2 \sqrt{-3}+\sqrt{-11}$ (resp. $v=5 \sqrt{-3}-2 \sqrt{-19}, v=-53 \sqrt{-3}-14 \sqrt{-43}$, $v=293 \sqrt{-3}+62 \sqrt{-67}, v=35573 \sqrt{-3}+4826 \sqrt{-163})$ est une unité fondamentale de $F$ pour $d=11$ (resp. pour $d=19, d=43, d=67, d=163$ ). Si l'on pose $t=v$ pour $d$ égal à $11,43,67$ et 163 , et $t=v^{3}$ pour $d$ égal à 19 , on vérifie aisément que $2 \pi t \equiv 1 \bmod (1-\omega)^{3}$. De cette congruence on déduit immédiatement que si $x=-\pi t$, alors $x$ est un générateur de $\mathfrak{p}$ tel que $x \equiv 1 \bmod 3$. On remarque que $2 p t$ et $x$ sont des nombres réels. Soit $\alpha$ la seul racine cubique réelle de $2 \pi t$; alors la proposition 1.4 assure que $a=(1 / 3)\left(1+\alpha+\left(\alpha^{2} / x\right)\right)$ engendre une base normale de $\mathfrak{O}_{E}$ sur $\mathfrak{O}_{F}$.

3. Descente à $K$. On démontre maintenant le théorème annoncé dans l'introduction. 
ThÉorème. Les racines du polynôme $X^{3}-X^{2}+X+1$ (resp. $X^{3}-X^{2}+$ $\left.X-163, X^{3}-X^{2}+X-447, X^{3}-X^{2}+X+663, X^{3}-X^{2}+X-15\right)$ constituent une base normale de $\mathfrak{O}_{K(2)}$ sur $\mathfrak{O}_{K}$ pour $K=\mathbb{Q}(\sqrt{-11})$ (resp. $K=\mathbb{Q}(\sqrt{-19}), K=\mathbb{Q}(\sqrt{-43}), K=\mathbb{Q}(\sqrt{-67}), K=\mathbb{Q}(\sqrt{-163}))$.

Soit $a=(1 / 3)\left(1+\alpha+\left(\alpha^{2} / x\right)\right)$ l'élément de $\mathfrak{O}_{E}$ qui a été calculé dans la démonstration de la proposition 2.1, c'est-à-dire, $\alpha$ est la seule racine cubique réelle de $2 \pi t$ et $x=-\pi t$; on sait que $a$ engendre une base normale de $\mathfrak{O}_{E}$ sur $\mathfrak{O}_{F}$. On veut démontrer que $a$ engendre une base normale de $\mathfrak{O}_{K(2)}$ sur $\mathfrak{O}_{K}$; d'après la proposition 1.3, il suffit de montrer que $a \in K(2)$.

Pour calculer le polynôme irréductible $P(X)$ de $a$ sur $K$, il suffit de considérer les conjugués de $a$ sous l'action de $\operatorname{Gal}(K(2) / K)$. Posons $\beta=$ $\alpha^{2} / x ;$ alors

$a=(1 / 3)(1+\alpha+\beta), \quad b=(1 / 3)\left(1+\omega \alpha+\omega^{2} \beta\right), \quad c=(1 / 3)\left(1+\omega^{2} \alpha+\omega \beta\right)$ sont les racines de $P(X)$ et

$$
P(X)=X^{3}-(a+b+c) X^{2}+(a b+a c+b c) X-a b c .
$$

On a

$$
\begin{gathered}
a+b+c=1, \quad a b+a c+b c=(1 / 3)(1-\alpha \beta), \\
a b c=(1 / 27)\left(1-3 \alpha \beta+\alpha^{3}+\beta^{3}\right) .
\end{gathered}
$$

On a $\alpha \beta=-2$ et $a b c$ égal à -1 (resp. $163,447,-663,15)$ pour $d=11$ (resp. $d=19, d=43, d=67, d=163)$. On a donc

$$
P(X)=X^{3}-X^{2}+X-\lambda,
$$

avec $\lambda=-1$ (resp. $\lambda=163, \lambda=447, \lambda=-663, \lambda=15$ ) pour $d=11$ (resp. $d=19, d=43, d=67, d=163)$. Le discriminant $\Delta$ de $P(X)$ est égal à $2^{2}(-11)\left(\right.$ resp. $2^{2} 97^{2}(-19), 2^{2} 3^{2} 59^{2}(-43), 2^{2} 3^{2} 67^{2}(-67), 2^{2} 3^{2}(-163)$ ) pour $d=11$ (resp. $d=19, d=43, d=67, d=163$ ). Ce fait implique que les racines de $P(X)$ engendrent un corps $L$ de degré 6 sur $\mathbb{Q}$ tel que $\operatorname{Gal}(L / \mathbb{Q})$ est isomorphe au groupe diédrale $D_{3}$ et $K$ est la seule extension quadratique de $\mathbb{Q}$ contenue dans $L$. On a $L \subseteq E$, l'extension $E / K$ est cyclique de degré six et $[L: K]=3$; on a donc $L=K(2)$. Par conséquent, $a \in K(2)$ et $a$ engendre une base normale de $\mathfrak{O}_{K(2)}$ sur $\mathfrak{O}_{K}$.

Remarque. Le corps $K(2)$ est le corps de décomposition de $P(X)$ sur $\mathbb{Q}$; on ne peut pas donc obtenir l'extension $(K(2) / K)$ comme la translation d'une extension galoisienne de $\mathbb{Q}$. Le corps $K(2)$ est une extension diédrale de $\mathbb{Q}$, de groupe de Galois $D_{3}$; J. Martinet et J.-J. Payan ont étudié ce type d'extensions dans leur article [9].

4. Construction d'un générateur avec des fonctions modulaires. Il est sans doute intéressant d'exprimer un générateur de base normale de 
$\mathfrak{O}_{K(2)}$ sur $\mathfrak{O}_{K}$ comme la valeur d'une fonction elliptique ou modulaire; autrement dit, d'exprimer avec des fonctions elliptiques ou modulaires un $\xi \in \mathfrak{O}_{K(2)}$ tel que

$$
\begin{gathered}
\operatorname{Tr}_{K(2) / K}(\xi) \in \mathfrak{O}_{K}^{*}=\{ \pm 1\}, \\
\left(\xi+\omega \xi^{\sigma}+\omega^{2} \xi^{\sigma^{2}}\right)^{3} \mathfrak{O}_{K}=\mathfrak{p}^{2} \mathfrak{q}, \\
\left(\xi+\omega^{2} \xi^{\sigma}+\omega \xi^{\sigma^{2}}\right)^{3} \mathfrak{O}_{K}=\mathfrak{p q}^{2},
\end{gathered}
$$

où $\operatorname{Gal}(K(2) / K)=\left\{1, \sigma, \sigma^{2}\right\}$. Un tel $\xi$ joue pour l'extension $K(2) / K$, du point de vue de la structure galoisienne, le même rôle que joue $\zeta_{p}=$ $\exp (2 \pi i / p)$ pour l'extension cyclotomique $\left(\mathbb{Q}\left(\zeta_{p}\right) / \mathbb{Q}\right)$.

Après avoir démontré le théorème principal contenu dans cet article, R. Schertz m'a montré à Augsburg les calculs qu'il avait réalisés il y a quelques années en utilisant les Klasseninvarianten de Weber ([11]), qu'on reproduit dans la suite.

Rappelons qu'on définit la fonction êta de Dedekind sur le demi-plan de Poincaré $\mathbb{H}$ par le produit infini

$$
\eta(\tau)=q_{\tau}^{1 / 24} \prod_{m=1}^{\infty}\left(1-q_{\tau}^{m}\right)
$$

avec $q_{\tau}=\exp (2 \pi i \tau)$; il s'agit donc d'une fonction holomorphe de $\mathbb{H}$ dans $\mathbb{C}$. On définit la fonction modulaire de Schläfli $f$ par

$$
f(\tau)=q_{\tau}^{1 / 48} \eta((\tau+1) / 2) / \eta(\tau)
$$

([11], p. 86 et p. 114; [1], $\S 6$; il faut noter que Birch, suivant Heegner, appelle $\sigma$ la fonction de Schläfli). La fonction de Schläfli est une fonction modulaire de niveau 48 définie dans un sous-groupe de $\Gamma(1)$ d'indice 72.

Soit $K=\mathbb{Q}(\sqrt{-d})$ avec $d=11,19,43,67$ ou 163 . On sait que $f(\sqrt{-d})$ est réel et possitif $([1]$, p. 291), que $K(2)=K(f(\sqrt{-d}))$ et que $f(\sqrt{-d})$ est un entier algébrique. On connait le polynôme irréductible $Q(X)$ de $f(\sqrt{-d})$ sur $K$; en fait, $f(\sqrt{-d})$ engendre une extension cubique non galoisienne de $\mathbb{Q}$ et $Q(X)$ est à coefficients entiers. On a $Q(X)$ égal à ([11], p. 475) :

$$
\begin{array}{ll}
X^{3}-2 X^{2}+2 X-2 & \text { pour } d=11, \\
X^{3}-2 X-2 & \text { pour } d=19, \\
X^{3}-2 X^{2}-2 & \text { pour } d=43, \\
X^{3}-2 X^{2}-2 X-2 & \text { pour } d=67, \\
X^{3}-6 X^{2}+4 X-2 & \text { pour } d=163 .
\end{array}
$$

Soit $\xi \in \mathfrak{O}_{K(2)}$ un entier de la forme

$$
\xi=u+x f(\sqrt{-d})+y f(\sqrt{-d})^{2}
$$


avec $u, x, y \in \mathbb{Z}$. Soit $\operatorname{Gal}(K(2) / K)=\left\{1, \sigma, \sigma^{2}\right\}, \omega=\exp (2 \pi i / 3)$ et

$$
\begin{aligned}
(\xi \mid 1) & =\xi+\xi^{\sigma}+\xi^{\sigma^{2}} \\
(\xi \mid \sigma) & =\xi+\omega \xi^{\sigma}+\omega^{2} \xi^{\sigma^{2}}, \\
\left(\xi \mid \sigma^{2}\right) & =\xi+\omega^{2} \xi^{\sigma}+\omega \xi^{\sigma^{2}},
\end{aligned}
$$

les trois résolvantes associées à $\operatorname{Gal}(K(2) / K)$ et construites sur $\xi$.

Appelons $\alpha=f(\sqrt{-d}), \beta=\alpha^{\sigma}$ et $\gamma=\alpha^{\sigma^{2}}$ les trois racines de $Q(X)$. Alors on a

$$
(\xi \mid \sigma)\left(\xi \mid \sigma^{2}\right)=r x^{2}+s x y+t y^{2},
$$

avec

$$
\begin{aligned}
& r=\alpha^{2}+\beta^{2}+\gamma^{2}-\alpha \beta-\alpha \gamma-\beta \gamma, \\
& s=2 \alpha^{3}+2 \beta^{3}+2 \gamma^{3}-\alpha \beta^{2}-\alpha \gamma^{2}-\beta \gamma^{2}-\alpha^{2} \beta-\alpha^{2} \gamma-\beta^{2} \gamma, \\
& t=\alpha^{4}+\beta^{4}+\gamma^{4}-\alpha^{2} \beta^{2}-\alpha^{2} \gamma^{2}-\beta^{2} \gamma^{2} .
\end{aligned}
$$

Les coefficients $r, s$ et $t$ sont des fonctions symétriques en $\alpha, \beta$ et $\gamma$, c'est-à-dire, des nombres entiers. Si l'on fait le calcul, on obtient

$$
(\xi \mid \sigma)\left(\xi \mid \sigma^{2}\right)=2 R(x, y)
$$

avec $R(x, y)$ égal à

$$
\begin{array}{ll}
-x^{2}+3 x y+6 y^{2} & \text { pour } d=11, \\
3 x^{2}+9 x y+2 y^{2} & \text { pour } d=19, \\
2 x^{2}+17 x y+20 y^{2} & \text { pour } d=43, \\
5 x^{2}+31 x y+38 y^{2} & \text { pour } d=67, \\
12 x^{2}+141 x y+404 y^{2} & \text { pour } d=163 .
\end{array}
$$

Supposons qu'on a trouvé un $(u, x, y) \in \mathbb{Z}^{3}$ tel que l'entier $\xi$ associé,

$$
\xi=u+x f(\sqrt{-d})+y f(\sqrt{-d})^{2},
$$

vérifie $(\xi \mid 1)= \pm 1$ et que $R(x, y)= \pm 1$. Puisque $D_{K(2) / K}=4 \mathfrak{O}_{K}$, on aura pour $\xi$ l'égalité

$$
\left((\xi \mid 1)^{2}(\xi \mid \sigma)^{2}\left(\xi \mid \sigma^{2}\right)^{2}\right) \mathfrak{O}_{K}=D_{K(2) / K}
$$

Mais

donc

$$
D_{K(2) / K}\left(\xi, \xi^{\sigma}, \xi^{\sigma^{2}}\right)=(\xi \mid 1)^{2}(\xi \mid \sigma)^{2}\left(\xi \mid \sigma^{2}\right)^{2},
$$

$$
D_{K(2) / K}\left(\xi, \xi^{\sigma}, \xi^{\sigma^{2}}\right)=D_{K(2) / K}
$$

et par conséquent $\xi$ engendre une base normale de $\mathfrak{O}_{K(2)}$ sur $\mathfrak{O}_{K}$.

Il est facile de trouver des couples $(x, y) \in \mathbb{Z}^{2}$ tels que $R(x, y)=-1$. Prenons, par exemple, $(x, y)$ égal à $(1,0)$ (resp. $(-1,4),(7,-1),(9,-2)$, $(5,-1))$ pour $d=11$ (resp. $d=19, d=43, d=67, d=163)$. 
On a

$$
(\xi \mid 1)=3 u+(\alpha+\beta+\gamma) x+\left(\alpha^{2}+\beta^{2}+\gamma^{2}\right) y .
$$

Puisque $\alpha+\beta+\gamma$ est égal à 2 (resp. $0,2,2,6$ ) pour $d=11$ (resp. $d=19$, $d=43, d=67, d=163)$ et $\alpha^{2}+\beta^{2}+\gamma^{2}$ est égal à $0($ resp. $4,4,8,28)$ pour $d=11$ (resp. $d=19, d=43, d=67, d=163$ ), si l'on considère le vecteur $(u, x, y)$ égal à $(-1,1,0)($ resp. $(-5,-1,4),(-3,7,-1),(-1,9,-2)$, $(-1,5,-1))$ pour $d=11$ (resp. $d=19, d=43, d=67, d=163$ ) et $\xi$ l'entier correspondant, on a $(\xi \mid 1)=1$ pour $d$ égal à 19 et 43 et $(\xi \mid 1)=-1$ pour $d$ égal à 11, 67 et 163 . On a donc montré le théorème suivant :

ThÉORÈme (Schertz). Soit $K=\mathbb{Q}(\sqrt{-d}), f$ la fonction modulaire de Schläfli et $\xi=u+x f(\sqrt{-d})+y f(\sqrt{-d})^{2}$ avec $(u, x, y)$ égal à $(-1,1,0)$ (resp. $(-5,-1,4),(-3,7,-1),(-1,9,-2),(-1,5,-1))$ pour $d=11$ (resp. $d=19, d=43, d=67, d=163)$. Alors $\xi$ engendre une base normale de $\mathfrak{O}_{K(2)}$ sur $\mathfrak{O}_{K}$ pour $d=11$ (resp. $\left.d=19, d=43, d=67, d=163\right)$.

Soit $j$ la fonction modulaire de Klein. On sait que $j(\sqrt{-d})$ est un entier pour $d$ égal à $11,19,43,67$ et 163 . Plus précisément, $j(\sqrt{-d})$ est égal à $-2^{15}$ (resp. $-2^{15} 3^{3},-2^{18} 3^{3} 5^{3},-2^{15} 3^{3} 5^{3} 11^{3},-2^{18} 3^{3} 5^{3} 23^{3} 29^{3}$ ) pour $d=11$ (resp. $d=19, d=43, d=67, d=163$ ). Grâce à la formule d'interpolation de Lagrange, il est possible de construire trois polynômes $R(X), S(X)$ et $T(X)$ à coefficients rationnels et de degré inférieur ou égal à quatre tels que le vecteur $(R(j(\sqrt{-d})), S(j(\sqrt{-d})), T(j(\sqrt{-d})))$ coïncide avec le vecteur $(u, x, y)$ du théorème précédent. Alors la fonction $g$ définie par

$$
g=R(j)+S(j) f+T(j) f^{2}
$$

est une fonction modulaire pour un sous-groupe $\Delta$ de $\Gamma(1)$ d'indice 72 qui contient $\Gamma(48)$ et $g(\sqrt{-d})$ est égale à la valeur $\xi$ du théorème pour les cinq valeurs de $d$ qui nous intéressent. On a donc le corollaire suivant.

Corollaire. Soit $K=\mathbb{Q}(\sqrt{-d})$ avec d égal à $11,19,43,67$ et 163 et soit $\tau \in \mathbb{H}$ tel que $\mathfrak{O}_{K}=\mathbb{Z} \tau+\mathbb{Z}$. Il existe une fonction g modulaire pour un sous-groupe $\Delta$ de $\Gamma(1)$ d'indice 72 qui contient $\Gamma(48)$ telle que $g(\tau)$ engendre une base normale de $\mathfrak{O}_{K(2)}$ sur $\mathfrak{O}_{K}$.

\section{Bibliographie}

[1] B. J. Birch, Weber's class invariants, Mathematika 16 (1969), 283-294.

[2] E. Brown and C. J. Parry, The imaginary bicyclic biquadratic fields with classnumber 1, J. Reine Angew. Math. 260 (1973), 118-120.

[3] E. J. Gómez Ayala, Bases normales d'entiers dans les extensions de Kummer de degré premier, J. Théor. Nombres Bordeaux 6 (1994), 95-116. 
[4] E. Hecke, Lectures on the Theory of Algebraic Numbers, Springer, New York, 1981.

[5] T. Kubota, Über den bizyklischen biquadratischen Zahlkörper, Nagoya Math. J. 10 (1956), 65-85.

[6] S. Lang, Algebraic Number Theory, Addison-Wesley, 1970.

[7] - Elliptic Functions, 2nd ed., Springer, New York, 1987.

[8] R. L. Long, Algebraic Number Theory, Marcel Dekker, New York, 1977.

[9] J. Martinet et J.-J. Payan, Sur les extensions cubiques non galoisiennes des rationnels et leur clôture galoisienne, J. Reine Angew. Math. 228 (1967), 15-37.

[10] R. Schertz, lettre à Ph. Cassou-Noguès du 4-2-91.

[11] H. Weber, Lehrbuch der Algebra, Bd. III, Braunschweig, 1908.

DEPARTAMENTO DE MATEMÁTICAS

FACULTAD DE CIENCIAS

UNIVERSIDAD DEL PAÍS VASCO

APARTADO 644

48080 BILBAO, ESPAÑA

E-mail: MTPGOAYE@LG.EHU.ES 